\title{
FERROMAGNETIC PROPERTIES OF ZnO:Mn NANOCRYSTALS OBTAINED BY THE FREEZE-DRYING METHOD
}

\author{
O.V. Kovalenko*, V.Yu. Vorovsky, O.V. Khmelenko, Ye.G. Plakhtii, O.I. Kushnerov \\ Oles Honchar Dnipro National University, Dnipro, Ukraine \\ *e-mail:kovalenko.dnu@gmail.com
}

In the work, the nanocrystals $\mathrm{ZnO}$ and $\mathrm{ZnO}$ :Mn with a concentration of $\mathrm{Mn} 2$ and 4 at.\% were obtained by the low-temperature freeze-drying method. For this purpose, solutions of zinc acetate $\mathrm{Zn}\left(\mathrm{CH}_{3} \mathrm{COO}\right)_{2} \cdot 2 \mathrm{H}_{2} \mathrm{O}$ and manganese one $\mathrm{Mn}\left(\mathrm{CH}_{3} \mathrm{COO}\right)_{2} \cdot 4 \mathrm{H}_{2} \mathrm{O}$ were used. By means of XRD, it is established that nanocrystals (NC's) have a pure phase and wurtzite-type hexagonal lattice, their size is $\mathrm{d} \sim$ $65 \mathrm{~nm}$. The EPR spectra of the samples have a broad absorption line. It is due to the presence of a large number of intrinsic and impurity defects in the NC's. These defects are the result of the destructive action of hydrogen, which is a product of the thermal decomposition of zinc and manganese acetates. It is shown that there is a relationship between the number of crystal lattice defects in the ZnO:Mn NC's and their ferromagnetic properties at room temperature. Samples of $\mathrm{ZnO}$ :Mn with a concentration of Mn 2 and 4 at.\%. have a specific magnetization value of $M_{s}$ equal to 0.089 and $0.045 \mathrm{emu} / \mathrm{g}$, respectively. The results can have great potential in spintronic devices and spin-based electronics.

Keywords: freeze-drying method, EPR spectra, ZnO:Mn nanocrystals, intrinsic and impurity defects, ferromagnetic properties.

Received 25.06.2020; Received in revised form 25.08.2020; Accepted 04.09.2020

\section{Introduction}

Diluted magnetic semiconductors (DMS) have unique electrical, piezoelectric, optical, and magnetic properties. They can be used in the field of microelectronics and spintronics. To obtain DMS, it is necessary to dop a nonmagnetic nanocrystalline semiconductor with a small amount of transition metal impurity, which leads to the appearance of ferromagnetic properties (FP) in such nanocrystals (NC's) at a room temperature. The main problem of obtaining DMS is to achieve a high degree of homogeneity and purity of the initial product. From this point of view, the freeze-drying method (FDM) has significant advantages, since it allows mixing the components at the molecular level at the stage of preparing solutions, and also ensures high purity of the technological process.

The FDM is based on the thermal decomposition of salts of the initial components, which solutions were frozen previously in the form of small drops and were dried by the freeze-drying method [1]. It is known that high temperatures of DMS synthesis lead to the disappearance of FP in the samples. At the same time, the temperatures for the preparation of NC's by the FDM from inorganic precursors, as a rule, are rather high. Thus, when sulfates are used as initial components, the temperature of nanocrystal synthesis exceeds $850^{\circ} \mathrm{C}$, which leads to the formation of large NC's and, as a consequence, to the disappearance of FP in the samples. Therefore, it becomes necessary to search for other precursors for the lowtemperature synthesis of nanomaterials by the FDM. Possible precursors for low-temperature synthesis of NC's $\mathrm{ZnO}: \mathrm{Mn}$ can be zinc acetate $\mathrm{Zn}\left(\mathrm{CH}_{3} \mathrm{COO}\right)_{2} \cdot 2 \mathrm{H}_{2} \mathrm{O}$ and manganese one $\mathrm{Mn}\left(\mathrm{CH}_{3} \mathrm{COO}\right)_{2} \cdot 4 \mathrm{H}_{2} \mathrm{O}$, the temperature of thermal decomposition of which is $325-350^{\circ} \mathrm{C}$ [2].

The aim of this work was to obtain NC's $\mathrm{ZnO}: \mathrm{Mn}$ by low-temperature FDM and to study their physical properties.

\section{Materials and methods}

Samples of $\mathrm{ZnO}$ and $\mathrm{ZnO}: \mathrm{Mn}$ NC's with a concentration of $\mathrm{Mn} 2$ and 4 at.\% were synthesized by the FDM. For this purpose, a 5\% aqueous solution of zinc acetate was used, where an appropriate amount of manganese acetate was added. The solutions were sprayed into drops of size $\mathrm{d}=20 \div 100 \mu \mathrm{m}$, which were frozen in liquid nitrogen then. 
Drying the obtained cryogranules was performed in a sublimation unit during 16 hours [3]. The synthesis of NC's occurred during the thermal decomposition of dry salts of zinc and manganese acetates at $350^{\circ} \mathrm{C}$. The duration of thermal decomposition affects the size of NC's. In order to obtain a small size of $\mathrm{ZnO}: \mathrm{Mn} \mathrm{NC}$ 's, heat treatment was carried out briefly during $20 \mathrm{~min}$ in the air. The study of the crystal structure and phase composition of the samples was carried out by the method of X-ray phase analysis (XPA) on a DRON-2 diffractometer using Co $\mathrm{K}_{\alpha}$ radiation $\left(\lambda=1.7902 \mathrm{~A}^{\circ}\right)$. The EPR study of the samples was carried out with a RADIOPAN SE/X 2543 radiospectrometer. The magnetic properties of the samples were studied by vibration magnetometry.

\section{Results and discussion}

The results of studying a $\mathrm{ZnO}$ : Mn sample with a $\mathrm{Mn}$ concentration of 4 at\% by $\mathrm{XRD}$ are shown in Fig. 1a. It was found that $\mathrm{ZnO}$ : Mn NC have a hexagonal lattice of the wurtzite type (in accordance with the standard JCPDS map: 36-1451). On the given X-ray diffraction pattern, additional reflections of the synthesis products are absent. This indicates that the period of heat treatment $(20$ minutes) is sufficient for the complete decomposition of the starting components. During the research, the interplanar distance $\boldsymbol{d}_{(h k l)}$ was calculated using the Bragg equation:

$$
2 d_{(h k l)} \sin \theta=n \lambda
$$

where $\boldsymbol{d}_{(h k l)}$ is the interplanar distance; $h, k$, and $l$ are Miller indices; $\theta$ is a Bragg angle; $n$ is the order of the diffraction maximum; $\lambda$ is the wavelength of X-rays. The values of the lattice parameters $a$ and $c$ for materials were determined by the equation

$$
\frac{1}{d_{(h k l)}^{2}}=\frac{4}{3}\left[\frac{h^{2}+h k+k^{2}}{a^{2}}\right]+\frac{l^{2}}{a^{2}}
$$

The unit cell volume for the hexagonal system was calculated using the equation

$$
V=0,866 a^{2} c
$$

For the $\mathrm{ZnO}$ : Mn NC sample (4 at.\%), we calculated the crystal lattice parameters $a$, $c$, the average NC size $d$, and the unit cell volume $V$. The following results were obtained: $\boldsymbol{a}=3.236 \AA, \boldsymbol{c}=5.197 \AA, \boldsymbol{d}=65.3 \mathrm{~nm}, \boldsymbol{V}=47.129 \AA^{3}$. In this case, the unit cell volume $\boldsymbol{V}$ of the synthesized sample significantly differs from that of a single-crystal $\mathrm{ZnO}$, for which $V_{\mu}=47.589 \AA^{3}$.

A decrease in the unit cell volume $V$ is a consequence of a decrease in interplanar spacings $\boldsymbol{d}_{(\boldsymbol{h k l})}$, which manifests itself in a shift in the position of the reflections of the Xray diffraction pattern of the sample relative to single-crystal $\mathrm{ZnO}$ (according to the JCPDS card: 36-1451) (Fig. 1, b). The displacement occurs towards large angles 2Ө, therefore, in accordance with equation (1), the interplanar distances $\boldsymbol{d}_{(h k l)}$ decrease. The fact of a decrease in the volume of the unit cell $\boldsymbol{V}$ indicates that the crystal lattice is in a nonequilibrium state and has deformation stresses. A similar decrease in the crystal lattice parameters of $\mathrm{ZnO} \mathrm{NC}$ in comparison with single-crystal $\mathrm{ZnO}$ was observed in works $[4,5]$. As a rule, doping of $\mathrm{ZnO} \mathrm{NC}$ with $\mathrm{Mn}$ impurity leads to an increase in the unit cell volume $\boldsymbol{V}$ [6]. Therefore, the answer to the question why this does not happen in our case requires additional research.

The EPR spectra of the synthesized samples (Fig. 2, a) differ significantly from the known EPR spectra of ZnO:Mn NC's. They have a wide, asymmetric absorption line of high intensity. 

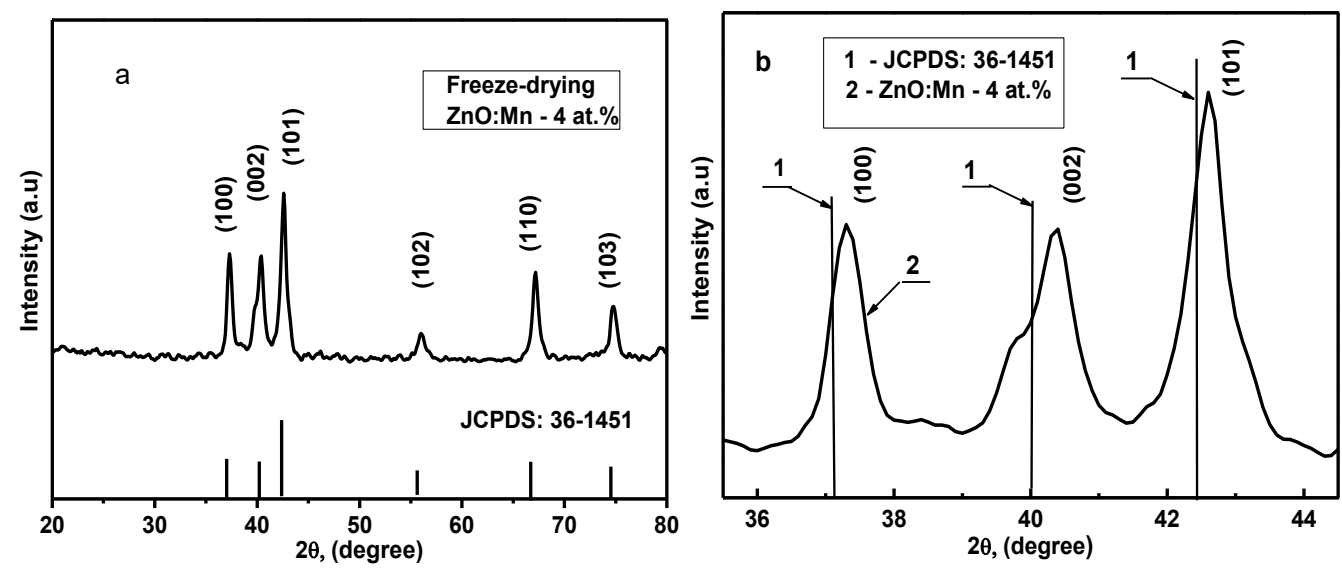

Fig. 1. Radiograph of the sample $\mathrm{ZnO}: \mathrm{Mn}$ (4 at.\%) (a) in comparison with the standard radiograph of monocrystalline $\mathrm{ZnO}$ type wurtzite (map JCPDS: 36-1451).

Offset of reflexes $(101),(002),(101)$ relative to standard positions (b).

In this case, the EPR spectrum of undoped $\mathrm{ZnO}$ is characterized by the absorption line of the highest intensity. An increase in the concentration of the Mn impurity leads to the decrease in the intensity of the broad absorption line and the appearance of signs of the presence of $\mathrm{Mn}^{2+}$ ions in the EPR spectrum of hyperfine structure (HFS) lines. This structure is conditional on $\mathrm{Mn}^{2+}$ ions, which are located in the nodes of the $\mathrm{ZnO}$ crystal lattice. The annealing of the sample $\mathrm{ZnO}: \mathrm{Mn}\left(4\right.$ at.\%) at $550^{\circ} \mathrm{C}$ in the air during 20 minutes also changes the structure of the EPR spectrum. It decreases the intensity of the main absorption line and increases the intensity of the HFS lines of $\mathrm{Mn}^{2+}$ ions. After annealing this sample at $850^{\circ} \mathrm{C}$ during 20 minutes in its EPR spectrum, the broad absorption line completely disappears leaving only the HFS lines of $\mathrm{Mn}^{2+}$ ions (Fig. 2, a, line 5).

Such transformation of the EPR spectra in $\mathrm{ZnO}$ and $\mathrm{ZnO}$ :Mn NC's is due to the redistribution in the synthesized samples of the concentration of intrinsic defects, such as interstitial zinc $\left(\mathrm{Zn}_{\mathfrak{f}}\right)$, oxygen vacancy $\left(\mathrm{V}_{\mathrm{o}}\right)$ and hydroxyl groups $-\mathrm{OH}$. In the samples of ZnO:Mn NC's, impurity defects are also added to these defects - interstitial manganese $\left(\mathrm{Mn}_{\mathrm{j}}\right)$ and $\mathrm{Mn}^{2+}$ ions, which replace $\mathrm{Zn}^{2+}$ ions in the $\mathrm{ZnO}$ lattice in isovalent way. All these defects are formed because of the destructive action of hydrogen, which is a product of thermal decomposition of zinc and manganese acetate [2]. Decomposition of zinc acetate carries out in two stages:

$$
\begin{gathered}
4 \mathrm{Zn}\left(\mathrm{CH}_{3} \mathrm{COO}\right)_{2}+\mathrm{H}_{2} \mathrm{O} \rightarrow \mathrm{Zn}_{4} \mathrm{O}\left(\mathrm{CH}_{3} \mathrm{COO}\right)_{6}+2 \mathrm{CH}_{3} \mathrm{COOH}, \\
\mathrm{Zn}_{4} \mathrm{O}\left(\mathrm{CH}_{3} \mathrm{COO}\right)_{6} \rightarrow 4 \mathrm{ZnO}+3 \mathrm{CH}_{3} \mathrm{COCH}_{3}+3 \mathrm{CO}_{2} .
\end{gathered}
$$

Acetone is obtained during this decomposition. Its decomposition leads to the appearance of hydrogen

$$
\mathrm{CH}_{3} \mathrm{COCH}_{3} \rightarrow \mathrm{C}_{2} \mathrm{H}_{4}+\mathrm{H}_{2} \uparrow+\mathrm{CO} \uparrow
$$

During long-term heat treatment of samples, due to the recrystallization of samples and their additional oxidation, the concentration of defects in the NC decreases, so it is possible to register only HFS $\mathrm{Mn}^{2+}$ ions in the EPR spectra. 


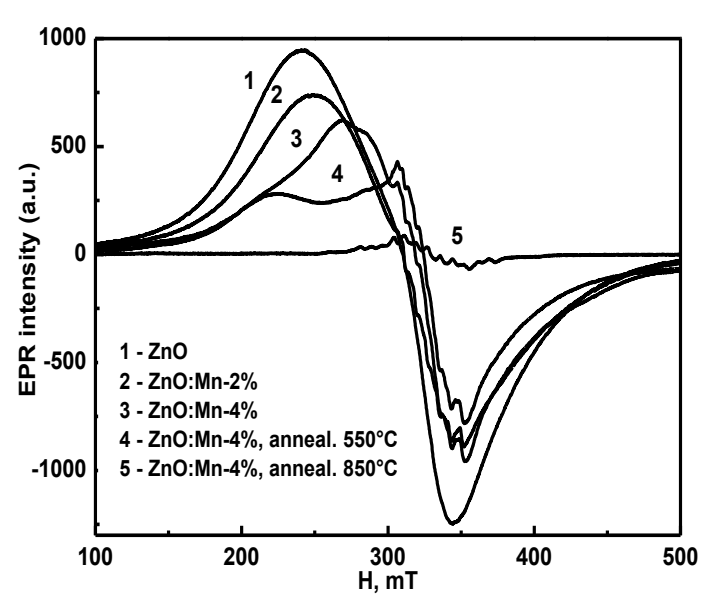

a

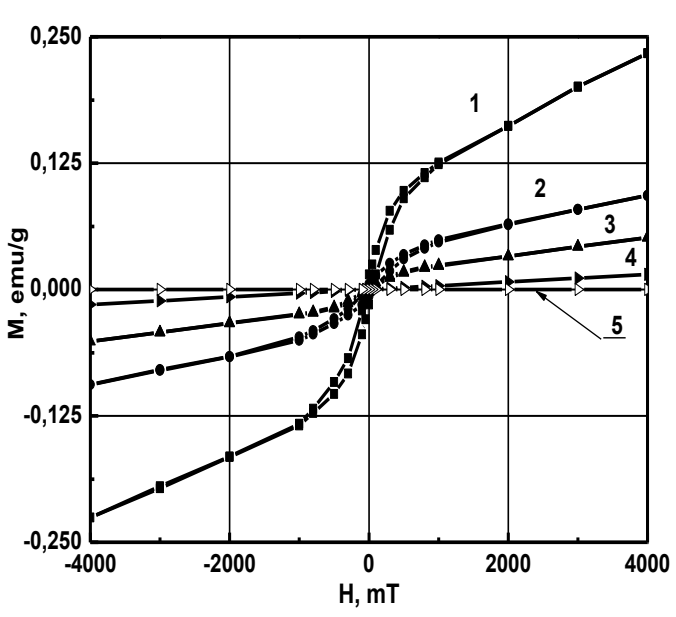

$\mathrm{b}$

Fig. 2 - EPR spectra (a) and magnetization curves (b) NC ZnO and ZnO: Mn: a - ZnO (1), ZnO:Mn with Mn concentration of 2 and 4 at.\% (2) and (3), respectively, (4) and (5) - sample (3) after annealing for 20 min at $550^{\circ} \mathrm{C}$ and $850^{\circ} \mathrm{C}$, respectively; magnetization curves $\mathrm{b}$ - $\mathrm{ZnO}$ : $\mathrm{Mn}$ with Mn concentration of 2 and 4 at.\% - (1) and (2), respectively, (3) and (4) - sample (2) after annealing for 20 min at $550^{\circ} \mathrm{C}$ and $850^{\circ} \mathrm{C}$, respectively, (5) - sample (2) after annealing for $1 \mathrm{~h}$ at $850^{\circ} \mathrm{C}$.

EPR spectra of various defects are observed in zinc oxide. Acceptor type defects have the value of the spectroscopic splitting factor $-\mathrm{g}$-factor of the EPR spectrum is greater than that of a free electron $g>g_{\alpha}\left(g_{\alpha}=2.00229\right)$. The EPR spectra of donor-type defects have a g-factor smaller than that of a free electron $g<g_{a}$.

The value of the spectroscopic splitting factor, the g-factor, was evaluated for all samples. The following results were obtained: $\mathrm{g}_{1}=2.249, \mathrm{~g}_{2}=2.215, \mathrm{~g}_{3}=2.140, \mathrm{~g}_{4}=$ $2.023, \mathrm{~g}_{5}=2.008$. Thus, due to the action of heat treatment there is decrease in the number of defects of the acceptor type in the samples, therefore the value of the g-factor of the EPR spectra is closer to the value for a free electron $\left(\mathrm{g}_{\mathrm{a}}=2.0023\right)$.

It is known that crystal lattice defects play a crucial role in the occurrence of FP in DMS. In the paper [7] it was shown that FP in ZnO:Mn NC's are due to the presence of intrinsic and impurity defects in them. According to the theoretical model of bound magnetic polarons [8], the magnetic interaction of these defects is the cause of the appearance of FP in samples at room temperature. In this case, intrinsic defects, such as oxygen vacancies $\left(\mathrm{V}_{\mathrm{o}}\right)$, act as intermediaries in the exchange interaction between impurity defects - interstitial Mn ions. Therefore, a decrease in the number of participants of the exchange interaction during the annealing ( $\mathrm{V}_{\mathrm{o}}$ vacancies and impurity $\mathrm{Mn}$ ions) should lead to a decrease in FP in the $\mathrm{ZnO}: \mathrm{Mn}$ NC's at room temperature. This conclusion is confirmed by our results (Fig. 2, b).

The absence of the saturation state on the magnetization curves of the samples indicates that in addition to the ferromagnetic phase there is also a paramagnetic phase, the number of which is determined by the angle of inclination of the lines tangent to these curves. The paramagnetic properties of the samples have a linear dependence on the magnetic field, and they disappear at zero magnetic field. Therefore, it is possible to isolate the ferromagnetic component from the experimental magnetization curves and determine the maximum specific magnetization of the samples in the saturation state of $M_{s}$ (Fig. 3, a). After such analysis, it was found that the specific magnetization $M_{s}$ of samples of ZnO:Mn NC's with Mn concentration - 2 and 4 at.\% is $0.094 \mathrm{emu} / \mathrm{g}$ and 
$0.045 \mathrm{emu} / \mathrm{g}$, respectively (Fig. 3, b). The obtained data of the magnetization of $M_{s}$ significantly exceed those in the samples of $\mathrm{ZnO}: \mathrm{Mn} \mathrm{NC}$ 's synthesized by the method of ultrasonic pyrolysis of the aerosol [9].

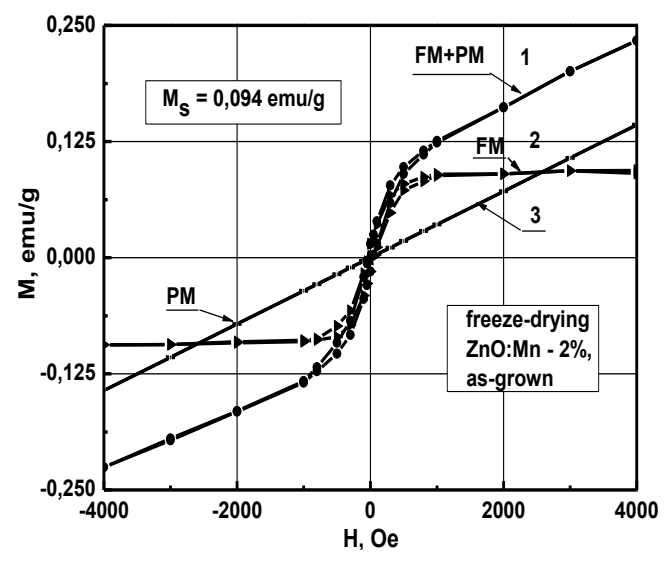

a

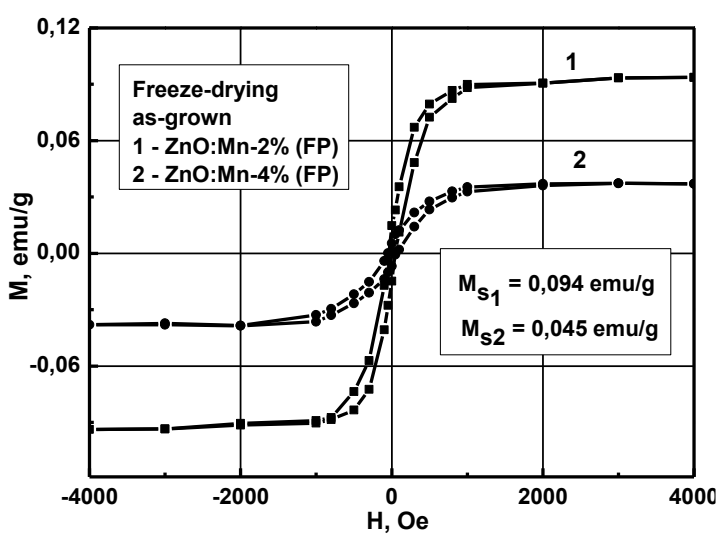

b

Fig. 3. The magnetization curve of the sample of $\mathrm{ZnO}: \mathrm{Mn}-2$ at. $\%$ (a): 1 - experimental curve, 2 ferromagnetic component of the magnetization of the sample (FM), 3 - paramagnetic component (PM).

Magnetization curves of ferromagnetic components of samples (b): $1-\mathrm{ZnO}: \mathrm{Mn} \mathrm{NC}-2$ at.\%, 2- ZnO:Mn NC -4 at.\%.

The decrease in the magnetization of $\mathrm{ZnO}: \mathrm{Mn}$ samples with increasing $\mathrm{Mn}$ concentration is explained by the fact that under such conditions some part of $\mathrm{Mn}^{2+}$ impurity ions can interact directly with each other due to the reduction of the distance. Theoretical calculations show that the samples partially acquire antiferromagnetic properties as a result of such interaction [8]. This leads to reduction of the magnetization of the samples.

Annealing of the sample in the air at $550^{\circ} \mathrm{C}$ also leads to a decrease in the value of the specific magnetization due to a decrease in the number of oxygen vacancies $\mathrm{V}_{\mathrm{o}}$. The sample after heat treatment at $850^{\circ} \mathrm{C}(20 \mathrm{~min})$ becomes completely paramagnetic (Fig. 2, b, curve 4 ) because oxygen vacancies $V_{o}$ completely disappear in such conditions, but some amount of impurity defects, which have paramagnetic properties, (Mn ions) remains. Annealing of the sample for 1 hour leads to complete loss of magnetic properties (Fig. 2, b, curve 5). This may be due to the absence in the sample of both intrinsic (oxygen vacancy $\mathrm{V}_{\mathrm{o}}$ ) and impurity (internodal Mn ions) defects of the crystal lattice which are necessary for the occurrence of exchange interaction.

\section{Conclusions}

The paper shows the possibility of obtaining ZnO:Mn NC's, which have ferromagnetic properties at room temperature, with low-temperature FDM. The main modes of synthesis were obtained, namely: impurity concentration $\mathrm{Mn}-2$ at.\%, synthesis temperature $-350^{\circ} \mathrm{C}$, synthesis duration $-20 \mathrm{~min}$. It is shown that the crystal lattice of $\mathrm{ZnO}: \mathrm{Mn} \mathrm{NC}$ is in the nonequilibrium state and has deformation stresses. The EPR method has revealed that the synthesized samples have a large number of acceptor-type defects. It is shown that there is a connection between the number of defects of the crystal lattice of $\mathrm{ZnO}: \mathrm{Mn} \mathrm{NC}$ 's and their ferromagnetic properties at room temperature. It is assumed that the peculiarity of the structure of the EPR spectra and the magnetic properties of the samples can be explained by the fact that during the synthesis of ZnO:Mn NC's, they are formed in a gaseous medium which contains hydrogen. It is a 
product of thermal decomposition of zinc and manganese acetates and provokes the formation of a large number of defects, which in their turn cause the ferromagnetism of $\mathrm{ZnO}: \mathrm{Mn}$ at room temperature.

The obtained results have both fundamental and applied significance. They can be used to explain the nature of ferromagnetism in DMS by searching those factors that are key in the formation of their magnetic properties. Also, these results can be valuable in creating a new generation of spintronics devices that use a spin degree of freedom along with a charger.

\section{References}

1. Generalov, M.B. Cryochemical nanotechnology. - Moscow: Akademkniga, 2006.

2. Anil,V.G. Simultaneous thermogravimetric analysis and in situ thermo-Raman spectroscopic investigation of thermal decomposition of zinc acetate dehydrate forming zinc oxide nanoparticles / V.G. Anil, L. Bertrand, T. Shin-Hwa, G. Kalyani, C. Hua, C.L. Yong // Chemical Physics Latters. - 2003. - Vol. 381. - P. 262 - 270.

3. Bulanyi, M. F. Structure of granules magnetic nanocrystals nickel-zinc ferrite obtained by cryochemical method / M.F. Bulanyi, V.Yu Vorovsky, A.V. Kovalenko, O.V. Khmelenko // J. Nano-Electron. Phys. - 2017. - Vol. 9, No. 3. - P. 36-41.

4. Kalita A. Size dependence of lattice parameters in $\mathrm{ZnO}$ nanocrystals_/ A. Kalita, M.P.C. Kalita // J. Applied Physics A. - 2015. - Vol. 121, No. 2. - P. $521-524$.

5. Yogamalar, R. X-ray peak broadening analysis in $\mathrm{ZnO}$ nanoparticles / R. Yogamalar, R. Srinivasan, A. Vinu, K. Ariga, A.C. Bose // J. Solid State Communications. - 2009. - Vol. 149. - P. $36-41$.

6. Sagar, R.V. Synthesis and magnetic behaviour of $\mathrm{Mn}: \mathrm{ZnO}$ nanocrystalline powders / R.V. Sagar, S. Buddhudu // Spectrochim. Acta A: Mol. Biomol. Spectrosc. 2010. - Vol. 75. - P. $1218-1222$.

7. Chattopadhyay, S. Defects induced ferromagnetism in $\mathrm{Mn}$ doped $\mathrm{ZnO} /$ S. Chattopadhyay, S.K. Neogi, A. Sarkar, M.D. Mukadam, S.M. Yusuf, A. Banerjee, S. Bandyopadhyay // J. Magn. Mater. - 2011. - Vol. 323 - P. 363 - 368.

8. Coey, J. M. D. Donor Impurity Band Exchange in Dilute Ferromagnetic Oxides / J.M.D. Coey, M. Venkatesan, C.B. Fitzgerald // J. Nature Mater. - 2005. - Vol. 4 - P. 173-179.

9. Vorovsky, V.Y. Preparation of zincoxide nanopowders doped with manganese which have ferromagnetic properties at room temperature / V.Y. Vorovsky, A.V. Kovalenko, A.I. Kushneryov, O.V. Khmelenko // J. Functional Materials. - 2018. - Vol. 25, No. 1. - P. $61-66$. 